\title{
Correlation Neglect in Financial Decision-Making
}

\author{
Erik Eyster and Georg Weizsäcker*
}

November 21, 2010

\begin{abstract}
Good decision-making often requires people to perceive and handle a myriad of statistical correlations. Notably, optimal portfolio theory depends upon a sophisticated understanding of the correlation between financial assets. In this paper, we examine people's understanding of correlation using a sequence of portfolio-allocation problems and find it to be strongly imperfect. Our experiment uses pairs of portfolio-choice problems that have the same asset span-identical sets of attainable returns - and differ only in the assets' correlation. While any outcome-based theory of choice makes the same prediction across paired problems, the subjects behave very differently across pairs. We find evidence for correlation neglect-people treat correlated variables as uncorrelated - as well as for a form of " $1 / n$ heuristic" - people invest half of wealth in each of two available assets. (JEL B49)
\end{abstract}

Keywords: portfolio choice, correlation neglect, $1 / n$ heuristic, biases in beliefs

${ }^{*}$ Eyster: Department of Economics, London School of Economics (email: e.eyster@lse.ac.uk); Weizsäcker: DIW Berlin \& Department of Economics, University College London \& Department of Economics, London School of Economics (email: gweizsaecker@diw.de). We are grateful to Xavier Gabaix, Jacob King, Matthew Rabin, Sven Rady, Tobias Schmidt, Paul Viefers and audiences at Berkeley, DIW Berlin, Bonn, East Anglia, Innsbruck, Toulouse and UCL, as well as the Second Behavioral Economics Annual Meeting (BEAM) at Cornell for their comments and to Jacob King and Wei Min Wang for superb research assistance. Both authors thank the STICERD research center at LSE for funding the experiments, Technical University Berlin for access to the decision laboratory, and the Schweizerische Nationalbank for funding the research opportunities at the ESSET 2008 conference. Weizsäcker thanks the ELSE Centre at UCL for financial support, and Eyster thanks the NIH for travel support. 


\section{Introduction}

Financial decision-makers face a panoply of correlations across different asset returns. Yet people have limited attention and find it cognitively challenging to work with joint distributions of multiple random variables. Even if in principle the typical investor could analyse financial variables' comovement adequately, she still might fail to account for correlation properly at the moment of allocating her financial portfolio. As a consequence, investors may hold portfolios that contain undesirable and avoidable risks. For example, many household investors invest disproportionately in stock of their own employers (Benartzi and Thaler 2001) or hold only a handful of positivelycorrelated assets (Polkovnichenko 2005). Following the recent financial-markets crisis, numerous commentators have asked whether both households and institutional investors relied on deficient risk modelling. ${ }^{1}$

In this paper, we explore people's tendency to neglect correlation. Although such "correlation neglect" may play an important role in numerous economic settings, we focus entirely on its consequences for financial decision-making. Many of the investment behaviours described above are broadly consistent with correlation neglect-but also with a multitude of other forces. To eliminate such confounds, we design and run a series of controlled experiments that test people's attention to correlation. Our experiment studies the standard textbook model of portfolio choice with statedependent returns using a framing variation in which each participant faces two versions of the same portfolio-choice problem. Across the two framing variations, we switch asset correlation on and off. This design thus ensures that under the null hypothesis that people correctly perceive the covariance structure, the framing variation does not affect their behavior. Nevertheless, we find that behavior changes strongly and the data analysis supports two alternative hypotheses, both irresponsive to correlation. First, people tend to ignore correlation and treat correlated assets as independent. Second, people tend to follow a simple $1 / \mathrm{n} "$ heuristic that prescribes investing equal shares of a financial portfolio into all available assets (as in Benartzi and Thaler (2001)). Our experimental data support these theories despite the fact that, through an understanding test, we ensure that the participants understand the payoff structure, including the co-movement of the

\footnotetext{
${ }^{1}$ Brunnermeier (2009) and Hellwig (2009) discuss erroneous perceptions of systemic risks during the crisis.
} 
asset returns. Consistent with limited attention, subjects appear to omit these considerations when choosing their portfolios.

A small set of previous experiments examining people's responses to correlation uncovers evidence consistent with neglect. Kroll, Levy and Rapoport (1988) and Kallir and Sonsino (2009) find that changing the correlation structure of a portfolio-choice problem leads to little or no change in participants' decision-making, even when many expected-utility preferences common in the economics and finance literatures predict significant change. Correlation neglect predicts no change in participants' behavior, consistent with the data. ${ }^{2}$ Our design reverses the previous ones: whereas Kroll et al. (1988) and Kallir and Sonsino (2009) vary the decision problems and find behavior unchanged, we keep the decision problems economically unchanged and find that behavior changes. These two approaches complement each other and together paint a consistent picture of correlation neglect. ${ }^{3}$

Our experimental design sharpens the findings of this past work because it allows us to test the null hypothesis that people correctly appreciate correlation without making any ancillary assumptions about subjects' utility functions. Chiefly, we need not assume that subjects are risk averse to test the null hypothesis that subjects correctly appreciate correlation. The reasons for this is that any fully rational agent will choose the same distribution of earnings in both isomorphic investment problems because the set of available portfolios is identical between them. This isomorphy arises through a straightforward manipulation: the assets in the correlated frame are linear combinations of the assets in the uncorrelated frame and thus span the exact same set of earnings distributions. ${ }^{4}$ Section 2 present the main experiment, which involves four such pairs of problems. In two of the problems, the correlated frame involves a positive correlation across assets, and in the other

\footnotetext{
${ }^{2}$ Kroll et al. (1988) also report evidence that choices respond remarkably little to feedback on realized returns. Kallir and Sonsino (2009) shed additional light on the cognitive nature of the bias that is consistent with the interpretation of correlation neglect as deriving from limited attention: when asked to predict one asset's return conditional upon the other's return, subjects demonstrate that they do perceive correlation correctly despite making investment choices that do not incorporate this understanding.

${ }^{3}$ Further closely related evidence is provided by Gubaydullina and Spiwoks (2009), whose subjects fail to minimize portfolio variance in a problem with correlated assets, but not in a different problem without correlation.

${ }^{4}$ This statement is modulo a qualifier about necessary short-sales constraints, which we clarify in Section 2.
} 
two problems it involves a negative correlation. The set of problems with correlated assets thus offers a nontrivial range of hedging opportunities, which may or may not be appreciated by the participants. ${ }^{5}$

Section 3 describes the theoretical predictions for these choice problems, based on a sequence of assumptions. For the standard, "rational" benchmark we first impose the most basic assumption that people have rational preferences over portfolios that depend only upon the distributions of their monetary payoffs. This consequentialist assumption - encompassing the entire set of expectedutility preferences as well as many generalizations in the literature-implies that if the decisionmaker were to make only one choice in our experiment, it would not matter which of the two correlation frames she faced. To invoke this implication in the data analysis, we make a second mild assumption ensuring that the decision-maker considers her choices in isolation: we assume that she either obeys the independence axiom or that she brackets narrowly between her choices (as defined in Section 3). Jointly, the stated assumptions in our experimental design enable us to reject of the null hypothesis that subjects correctly perceive correlation, regardless of their risk attitudes. A further (and standard) assumption that we use for some purposes is that the decisionmaker is risk averse. Risk aversion suffices to make a unique prediction in three of our four pairs of problems that have a unique portfolio that second-order stochastically dominates all others. For the remaining pair, we use the stronger assumption that the (risk-averse) decision-maker is close to being risk neutral to make a unique prediction.

The same full set of of assumptions suffices to make a unique prediction in each choice problem for a decision-maker who fully neglects correlation and treats all random variables as independent. To model correlation neglect, we begin with both assets' marginal distribution over payoffs and construct their product distribution over payoffs; someone who neglects correlation misperceives payoffs as deriving from this product distribution - where, by construction, the assets' payoffs are uncorrelated - rather than from the true conditional distribution. Finally, Section 3 also discusses the third behavioral model that we consider, a decision-maker who simply invests equal proportions

\footnotetext{
${ }^{5}$ A notable feature of our design is its minimality: switching correlation between non-degenerate random variables on and off requires at least as many assets (two) states of nature (four) and distinct monetary prizes (three) as we employ. In this sense, correlation neglect may appear in the simplest possible set-up.
} 
of her wealth in each available asset. As will become clear, this may be viewed as an extreme version of "variance neglect", where the decision-maker ignores differences in the asset variances.

The data summary of Section 4 shows that very few participants choose equivalent portfolios in paired choice problems. Only one out of 146 participants in the main experiment behaves fully consistently, choosing four equivalent portfolios in the four pairs of choices. Of the remaining participants, a majority (60\%) does not choose even a single isomorphic pair of portfolios in any of the four pairs of choices. A surprising result appears regarding the relative predictive value of the three basic models (rational, perceived independence, $1 / \mathrm{n}$ ). In linear regressions, the rational model adds no explanatory power to the other two. Regressing subjects' choices in the correlated problems on the predictions of the first two models, the rational model has a point estimate with the wrong sign.

Section 5 further examines the patterns in subjects' deviations from rationality. There again we exploit the fact that all assets pay the same expected return to decompose subjects' preferences over portfolios into preferences over the variance in payoffs. Thus retaining the assumption of meanvariance preferences, we allow for a wider set of erroneous perceptions of the covariance structure. ${ }^{6}$ First, participants' perceptions of correlation may be biased towards zero. Second, they may underestimate the magnitude of differences between the entries of the variance-covariance matrix by using a concave transformation of variance. We classify subjects into types according to two parameters, one measuring correlation neglect and the other variance neglect. The single type that best fits the entire subject pool is one that entirely neglects correlation and exponentiates variance to the power 0.43 . The two types that best fit the subject pool are one covering ninety-one percent of subjects that essentially coincides with that just described and a second, rational type best fitting the remaining nine percent of subjects. Adding additional types does not significantly improve the statistical fit. These estimations indicate that substantial proportions of the participants follow the extreme cases of these two biases - they either neglect all covariance or all variance differences - or show combinations of the biases. Under the simplifying assumption that all participants either correctly appreciate the covariance structure or show one of the two extreme biases, we find that

\footnotetext{
${ }^{6}$ The result in Eyster (2010) clarifies that mild risk aversion leads to approximate mean-variance preferences.
} 
an estimated $10 \%$ of the participants show the correct appreciation of the distribution.

Our study also includes a few more experimental demonstrations of correlation neglect, which we present in Section 6. In one, we offer the participants two portfolios, the first riskier than the second, with the property that if one mistakenly ignores the correlation between the underlying assets the first portfolio appears to first-order stochastically dominate the second portfolio. Indeed, almost all subjects choose the apparently dominant option. But in a control treatment, where the the same two portfolios are offered but their true distributions are explicitly shown to the subjects, about half of the subjects choose the second option.

The final demonstration of correlation neglect is that participants can be made to violate arbitrage freeness. In a separate task we present the participants with three assets, where one asset is state-wise dominated by appropriate combinations of the other two. Any investment in the dominated asset is an arbitrage loss: spreading that investment appropriately across the other two assets would yield more money in every state of the world. We find that more than three quarters of our subjects indeed fall prey to this arbitrage. Section 7 concludes.

\section{Experimental Design and Procedure}

The following excerpt from the instructions shows one of the decision problems, labelled Choice 3.

3. Invest each of your 60 points in either Asset $\mathrm{E}$ or Asset F, as given below.

\begin{tabular}{ccccc} 
& {$[1]$} & {$[2]$} & {$[3]$} & {$[4]$} \\
\hline$E$ & 12 & 24 & 12 & 24 \\
$F$ & 12 & 12 & 24 & 24
\end{tabular}

Let $\Omega=\{1,2,3,4\}$ be the four equi-probable possible states of the world, denoted by columns in the table above. Each row's label $X$ denotes an asset that pays out $X(\omega)$ in state $\omega \in \Omega$. In Choice 3, for instance, Asset $E$ pays out $E(1)=12$ in state 1, $E(2)=24$ in state 2, etc. Each entry states how many Euros a subject who invested her entire portfolio in that asset would earn. The state $\omega$ is chosen through a random draw with equal probabilities across the four states in $\Omega$. Each participant faces $N=11$ or $N=12$ choice problems in this format, without feedback between choices. Of 
these, ten problems were presented in the first part of the experiment. The additional one or two choices, described in Section 6, followed after a brief additional instruction. Although the content of this additional instruction varied among participants, its placement after the first ten problems prevented it from influencing the initial ten choices. We focus on eight of the initial ten choices in this section, which constitute the main experiment; the remaining two choices are reported in Appendix B. ${ }^{7}$ Only one of the $N$ choices of the experiment is paid out for each subject, following another random draw that each subject makes at the conclusion of the experiment. ${ }^{8}$ Given this random payment procedure, participants can be assumed to make each choice in isolation given preferences that satisfy the Independence Axiom or given that she "brackets narrowly". We return to this issue in Section 3.

The set of available portfolios in decision problem $n \in\{1, \ldots, N\}$ is characterized by the two available assets $X_{1}^{n}$ and $X_{2}^{n}$. Any portfolio can be viewed as a lottery over wealth $W$ that assigns wealth $W(\omega)$ to state $\omega$ : a decision maker who invests the fraction $\alpha_{1}^{n}$ of her wealth $\left(60 \cdot \alpha_{1}^{n}\right.$ "points") into asset $X_{1}^{n}$ and the remaining fraction $1-\alpha_{1}^{n}$ into asset $X_{2}^{n}$ ends with wealth $W(\omega)=\alpha_{1}^{n} X_{1}^{n}(\omega)+\left(1-\alpha_{1}^{n}\right) X_{2}^{n}(\omega)$ in state $\omega .^{9} \alpha_{1}^{n}$ also must lie in some constraint set $\mathcal{C}^{n} \subset$ $\mathbb{R}$, which precludes short sales of either asset, $0 \leq \alpha_{1}^{n} \leq 1$, and in some cases includes more stringent constraints. The following table shows our main 8 choice problems by reproducing the 8 specifications of $\left(X_{1}^{n}, X_{2}^{n} ; \mathcal{C}^{n}\right)$ for $n=1, \ldots 8$. The experiment presented the problems in two different sequences, used abstract labels for states, and varied the order in which states were presented.

\footnotetext{
${ }^{7}$ The two remaining choices exactly resemble those discussed in the main text and were designed to demonstrate that correlation neglect can lead to violations of blatantly obvious statewise dominance between assets. Appendix B reports a statistically significant (but economically small) effect of this nature.

${ }^{8}$ In addition, participants receive a show-up fee of 5 Euros.

${ }^{9}$ Although the participants were required to choose integer point allocations, putting $\alpha_{1}^{n}$ onto a grid, we ignore this complication for simplicity.
} 


\begin{tabular}{ccc}
\hline \hline$n$ & $\left\{X_{1}^{n}(1), X_{1}^{n}(2), X_{1}^{n}(3), X_{1}^{n}(4)\right\}\left\{X_{2}^{n}(1), X_{2}^{n}(2), X_{2}^{n}(3), X_{2}^{n}(4)\right\}$ & $\mathcal{C}^{n}$ \\
\hline 1 & $\{15,21,15,21\}\{12,12,24,24\}$ & $0 \leq \alpha_{1}^{1} \leq 1$ \\
2 & $\{18,30,6,18\}\{12,12,24,24\}$ & $0 \leq \alpha_{1}^{2} \leq \frac{1}{2}$ \\
3 & $\{12,24,12,24\}\{12,12,24,24\}$ & $0 \leq \alpha_{1}^{3} \leq 1$ \\
4 & $\{12,24,12,24\}\{12,18,18,24\}$ & $0 \leq \alpha_{1}^{4} \leq 1$ \\
5 & $\{14,21,14,21\}\{14,14,21,21\}$ & $0 \leq \alpha_{1}^{5} \leq 1$ \\
6 & $\{14,21,14,21\}\{14,0,35,21\}$ & $\frac{2}{3} \leq \alpha_{1}^{6} \leq 1$ \\
7 & $\{12,30,12,30\}\{12,12,30,30\}$ & $0 \leq \alpha_{1}^{7} \leq 1$ \\
8 & $\{12,30,12,30\}\{12,18,24,30\}$ & $0 \leq \alpha_{1}^{8} \leq 1$ \\
\hline
\end{tabular}

Table 1: 8 portfolio-choice problems.

An important feature of the 8 problems is that half - those with odd-numbered $n$-involve only pairs of uncorrelated assets, whereas the other half involve non-zero correlations. In Section 3, we explain how each even-numbered decision problem is isomorphic to its immediate predecessor in the table.

The participants for the eight decision problems described above were 148 students of Technical University Berlin, mostly undergraduate. Of these, two participants violated one of the contraints $\mathcal{C}^{n}$ and their data were thus excluded from the analysis, leaving 146 complete observations. The eight experimental sessions were conducted in a paper-and-pencil format (with instructions translated into German) following a fixed protocol and with the same experimenters present. Each session lasted about 90 minutes, including all payments. Before the main part of the experiment, the participants underwent an understanding test asking three questions about the payment rule, all of which subjects had to answer correctly before proceeding. About $10 \%$ of the subjects needed help from the experimenters to pass the understanding test. Controlled variations of the eight decision problems were also used in four further sessions with 96 additional subjects (see Section $6)$. 


\section{Predictions}

In this section, we develop predictions in our experiments deriving from various different assumptions about the decision maker's choice functions, moving from weakest to strongest. Let $\mathcal{W}^{n}=\mathcal{W}\left(X_{1}^{n}, X_{2}^{n} ; \mathcal{C}^{n}\right)$ be the set of wealth lotteries feasible for the investor in decision problem $n$ Because subjects were only paid for a single choice chosen at random, we work with their preferences over $\mathcal{W}$, the feasible set of wealth lotteries across the entire experiment. ${ }^{10}$ For any $S \subset \mathcal{W}$, let $m_{\succeq}(S)=\left\{s \in S: \forall s^{\prime} \in S, s \succeq s^{\prime}\right\}$, the investor's set of preferred lotteries from $S$.

Assumption A. The investor makes choices that maximise a preference relation $\succeq$ over $\mathcal{W}$ that is complete and transitive. Moreover, for each $n, m_{\succeq}\left(\mathcal{W}^{n}\right)$ is a singleton set.

Assumption A implies that investors' preferences over asset portfolios are rational; they depend only on the span of assets and not the correlation structure underlying it. When different assets pay different expected returns, then the risk-return tradeoff normally produces non-singleton indifference curves. In our experiment, however, in each choice, each asset pays the same expected return. Because our design eliminates any risk-return tradeoff, it makes sense to assume that indifference curves are degenerate. Nevertheless, it suffices to assume-and that we do assume - that the decision maker has a unique preference-maximising portfolio in each of the choice sets of the experiment. Whenever two portfolio-allocation problems have the same asset span, any decision maker who makes choices to maximise rational preferences over wealth lotteries must make the same choice across both problems. In particular, if a subject were to make only one choice in the experiment, then it would only depend upon the span of assets in that choice.

Observation 1. Under Assumption $A$, if $\mathcal{W}\left(X_{1}^{\prime}, X_{2}^{\prime} ; \mathcal{C}^{\prime}\right)=\mathcal{W}\left(X_{1}, X_{2} ; \mathcal{C}\right)$, then $m_{\succeq}\left(\mathcal{W}\left(X_{1}^{\prime}, X_{2}^{\prime} ; \mathcal{C}^{\prime}\right)\right)=$ $m_{\succeq}\left(\mathcal{W}\left(X_{1}, X_{2} ; \mathcal{C}\right)\right)$.

This results holds not only for expected-utility preferences but for all rational preference relations, regardless of whether they are continuous or satisfy the independence axiom. In our experiment, twinned problems are constructed to have identical asset spans. For example, in Choices 3 and 4

\footnotetext{
${ }^{10}$ Since $\alpha_{1}^{n}$ lies on a grid in each choice problem $n$, the experiment offers only a finite number of achievable wealth levels. The set of probability distributions over these wealth levels contains $\mathcal{W}$.
} 
presented above in the text, Asset G is identical to Asset E, and Asset H equals $\frac{1}{2} E+\frac{1}{2} F$. Someone who invests $\hat{\alpha} \geq \frac{1}{2}$ in Asset $\mathrm{E}$ of Choice 3 can achieve the same portfolio with $\alpha=2 \hat{\alpha}-1$ in Asset $\mathrm{G}$ of Choice 4. This holds because the remaining $1-\alpha=1-(2 \hat{\alpha}-1)=2(1-\hat{\alpha})$ invested in Asset $\mathrm{H}$, itself comprised of $\frac{1}{2} E+\frac{1}{2} F$, gives $\frac{1}{2} 2(1-\hat{\alpha})=(1-\hat{\alpha})$ invested in Asset F, just like in Choice 3. Hence, any portfolio produced using $\hat{\alpha} \geq \frac{1}{2}$ in Choice 3 can be reproduced through a suitably constructed portfolio in Choice 4 , and vice versa. Although this argument breaks down for $\hat{\alpha}<\frac{1}{2}$, the symmetry of Choice 3 across assets and states, as well as our experiment's random presentation of states (columns are randomly permuted), suggests that anyone who wishes to invest less than one-half of her portfolio in Asset E, essentially betting on State 3 over the symmetric State 2, should be equally willing to invest more than one-half in Asset F. Similarly, the other three pairs of twinned problems have been constructed so that every feasible portfolio in the uncorrelated problem can be replicated in the correlated problem, and vice versa.

Observation 1 implies that if each subject in our experiment were to make only a single portfolioallocation choice, then she would take positions in twinned problems that result in the same statecontingent wealth lottery. However, each subject in our experiment does not make a single choice but instead a sequence of choices, with only a single choice randomly chosen to be paid out. In this case, we must also assume that the decision maker adheres to the independence axiom in order to conclude that she chooses the same portfolio across twinned problems. This limits the scope of the result to expected-utility preferences, be they risk-averse, risk-loving, or neither.

Yet Tversky and Kahneman (1981) and Rabin and Weizsäcker (2009), among others, demonstrate that subjects in experiments do not makes choices on individual problems that take into account the entire set of problems they have to solve - even when explicitly told to do so-but instead make each choice in isolation, neglecting all remaining problems. We capture this idea by a definition similar to that in Rabin and Weizsäcker (2009).

Definition 1. The decision maker brackets narrowly if she makes each portfolio-allocation choice as if it were her only one: she applies applies her preference relation to the choice set given by $\left(X_{1}^{n}, X_{2}^{n} ; \mathcal{C}^{n}\right)$

A subject who"brackets narrowly" in this sense chooses the same portfolio across twinned 
problems to conform to Observation 1 regardless of whether she obeys the Independence Axiom.

Assumption B. The decision maker brackets narrowly or makes choices to maximise preferences that satisfy the Independence Axiom.

Together Assumptions A and B imply Observation 1 in our experiment where only a single choice is actually paid out to subjects.

A standard assumption about preferences under uncertainty is that people dislike risk.

Assumption C. The decision maker is risk averse. ${ }^{11}$

Because each asset in our experiment pays out a positive amount in each state of the world, a subject who maximised Kahneman and Tversky (1979) loss-averse preferences over lab winnings - using a zero reference point- - satisfies Assumptions A-C.

In three out of four pairs of twinned problems (Choices 3-8), Assumptions A,B and C suffice to make unique predictions about subjects' choices. For instance, consider Choice 3, described in the last section, together with Choice 4 .

4. Invest each of your 60 points in either Asset G or Asset H, as given below.

\begin{tabular}{ccccc} 
& {$[1]$} & {$[2]$} & {$[3]$} & {$[4]$} \\
\hline$G$ & 12 & 24 & 12 & 24 \\
$H$ & 12 & 18 & 18 & 24
\end{tabular}

In both Choices 3 and 4, any portfolio leads to a payoff of 12 in state 1 and 24 in state 4 . In Problem 3, investing $\alpha_{E}$ in Asset $E$ and $1-\alpha_{E}$ in Asset $F$ gives payoffs $24 \alpha_{E}+12\left(1-\alpha_{E}\right)=12+12 \alpha_{E}$ in state 2 and $12 \alpha_{E}+24\left(1-\alpha_{E}\right)=24-12 \alpha_{E}$ in state 3 . Since states 2 and 3 occur with the same probability, any risk averter prefers to the expected value of her money payoff across the two states, 18 , in each state to any lottery. This can be achieved by choosing $\alpha_{E}=\frac{1}{2}$. Each of Choices 3 through 8 has the feature that in two states the decision maker can do nothing to hedge her risk while in the remaining two she can perfectly hedge her risk just as in this case. Since each twinned

\footnotetext{
${ }^{11}$ Formally, for every lottery $L$, the decision maker weakly prefers the degenerate lottery paying $E[L]$ with certainty to $L$.
} 
In Choices 1 and 2, risk aversion alone does not suffice to identify subjects' optimal choice. In this case, adding Assumption D suffices to make a unique prediction.

Assumption D. The decision maker is arbitrarily close to risk neutral. ${ }^{12}$

Observation 2. Under Assumptions $A-D$, for each $n, m_{\succeq}(\mathcal{W})$ minimises the variance in portfolio earnings in each choice $\mathcal{W}^{n}$.

Eyster (2010) contains a more general theorem along these lines and its proof. ${ }^{13}$ Intuitively, uniform arbitrary closeness to risk neutrality implies arbitrary closeness to constant-absolute risk aversion (CARA), which collapses to lexicographic mean-variance preferences as the decision maker approaches risk neutrality.

An alternative hypothesis is that our decision maker neglects the correlation in assets' returns, treating each asset as independent. This violates Assumption A because such a decision maker does not maximise rational preferences over state-contingent payoffs. We model a hexed decision maker as one who maximises rational preferences over a transformed, fictitious asset space where assets are uncorrelated. To construct this fictitious asset space, let

$$
\widehat{\Omega}=\{1.1,1.2,1.3,1.4,2.1,2.2,2.3,2.4,3.1,3.2,3.3,3.4,4.1,4.2,4.3,4.4\}
$$

and suppose that in state $a . b$, the first asset pays $X_{1}(a)$ and the second $X_{2}(b)$. Whereas $X_{1}, X_{2}$ : $\Omega \rightarrow \mathbb{R}$, these new assets $\widehat{X}_{1}, \widehat{X}_{2}: \widehat{\Omega} \rightarrow \mathbb{R}$. Most importantly, whatever the correlation between $X_{1}$ and $X_{2}, \widehat{X}_{1}$ is independent of $\widehat{X}_{2}$ by construction: whatever the payout of $\widehat{X}_{1}, \widehat{X}_{2}$ pays out $\widehat{X}_{2}(1), \widehat{X}_{2}(2), \widehat{X}_{2}(3)$ and $\widehat{X}_{2}(4)$ with equal probability.

Let $\widehat{\mathcal{W}}^{n}=\widehat{\mathcal{W}}\left(\widehat{X}_{1}^{n}, \widehat{X}_{2}^{n} ; \mathcal{C}^{n}\right)$ be the set of wealth lotteries feasible for the investor when the assets are $\widehat{X}_{1}^{n}, \widehat{X}_{2}^{n}$ given the constraints $\mathcal{C}^{n}$. Let $\widehat{\mathcal{W}}$ be the set of lotteries over wealth levels across the entire asset with the transformed assets $\left(\widehat{X}_{1}, \widehat{X}_{2}\right)$.

\footnotetext{
${ }^{12}$ Formally, consider a sequence of expected utility maximisers with $C^{2}$, concave Bernouilli utility functions $\left(u_{n}\right)_{n \in \mathbb{N}}$ and their associated Arrow-Pratt coefficient-of-absolute-risk aversion functions $\left(r_{n}\right)_{n \in \mathbb{N}}$. Let $\alpha_{n}$ denote the portfolio that maximises the expectation of $u_{n}$. When $\left(r_{n}\right)_{n \in \mathbb{N}}$ converges uniformly to zero, and the decision maker chooses a portfolio that belongs to the limit of $\left(\alpha_{n \in \mathbb{N}}\right)$, we say that the decision maker is arbitrarily close to risk neutral.

${ }^{13}$ Formally, $\left(\alpha_{n}\right)_{n \in \mathbb{N}}$ converges to the portfolio that maximises lexicographic preferences in the moments, with alternating sign. In the context of our experiment where assets have equal means and there is at most one portfolio with any given variance, this coincides with minimising portfolio variance.
} 
Assumption E. The decision maker makes choices that maximise a preference relation $\succeq$ over $\widehat{\mathcal{W}}$ that is complete and transitive. Moreover, for each $n, m_{\succeq}\left(\widehat{\mathcal{W}}^{n}\right)$ is a singleton set.

When $X_{1}$ and $X_{2}$ are uncorrelated, $\mathcal{W}$ is equivalent to $\widehat{\mathcal{W}}$, which implies that rational and hexed investors make the same choices.

Observation 3. When $X_{1}$ is independent of $X_{2}$, Assumptions $A$ and $B$ produce the same choices as do Assumptions $B$ and $E$.

When assets are correlated, Assumptions B,C,D, and E suffice to make a unique prediction for exactly the reasons that Assumptions A, B,C, and D do above. In particular, in each choice the investor allocates her portfolio to minimise her perceived portfolio variance in the original problem, which equals the actual variance in the hatted problem.

When $X_{1}$ is positively correlated with $X_{2}$, a hexed investor underestimates the variance in her portfolio. Let $\sigma_{12}$ be the covariance between $X_{1}$ and $X_{2}$.

Proposition 1. When $\sigma_{12}>0$, Assumptions $B, C, D$, and E give a (weakly) more equal split of portfolio across assets than Assumptions $A, B, C$, and D. When $\sigma_{12}<0$, Assumptions B, C,D, and E give a (weakly) more unequal split of portfolio across assets than Assumptions $A, B, C$, and D.

When assets are positively correlated, a hexed investor overestimates diversification benefit of moving his portfolio from a low-variance asset to a high variance one, which leads her to take a more highly diversified portfolio than a rational decision maker, a form of false diversification effect. When assets are negatively correlated, a hexer underestimates the diversification benefit of moving his portfolio from a low-variance asset to a high variance one, which leads her to take a less diversified portfolio than a rational decision maker, a form of hedging neglect.

Benartzi and Thaler (2001) have suggested that investors facing a menu of $n$ different mutual funds often use the simple heuristic of investing the fraction $\frac{1}{n}$ of her portfolio in each fund. In the context of our experiment, investing half the portfolio in each of the two choices would lead an investor to make different portfolios in paired problems.

We have seen that Assumptions A-D as well as B-E imply lexicographic mean-variance preferences, albeit with different perceived covariance matrices. In settings where people are close to risk 
neutral such that they maximise mean-variance preferences, we can consider a simple parametric alternative that incorportates diminishing sensitivity to variance of any kind. While much research has demonstrated people's aversion to risk - even at its smallest scale - that does not imply that they dislike increased risk as much as the independence axiom implies. Like Fechner and Weber famously demonstrated with physical stimuli, people's perception of or distaste for risk may increase slower than linearly with variance. We capture this possibility by positing that people maximise mean-variance preferences using a transformed covariance matrix as follows:

$$
V=\left(\begin{array}{cc}
\left(\sigma_{1}^{2}\right)^{l} & k \cdot \operatorname{sgn}\left(\sigma_{12}\right)\left|\sigma_{12}\right|^{l} \\
k \cdot \operatorname{sgn}\left(\sigma_{12}\right)\left|\sigma_{12}\right|^{l} & \left(\sigma_{2}^{2}\right)^{l}
\end{array}\right)
$$

where $k, l \in[0,1]$.

Using this transformed covariance matrix allows us to nest our several hypotheses about subject behaviour.

RAT: Subjects satisfy Assumptions A-D, i.e., $k=l=1$.

Hex: Subjects satisfy Assumptions B-E, i.e., $k=0$ and $l=1$.

1/N: Subjects use the $\frac{1}{N}$ heuristic, i.e., $l=0$.

Finally, when $k=0$ and $l<1$, behaviour conforms to a hexer who also exhibits diminishing sensitivity to variance.

\section{Evidence of Correlation Neglect: Raw Data and Analysis of Means}

This section shows the raw data of the experiment and tests whether Assumptions A and B are satisfied. The observed data patterns show evidence of both directions of correlation neglect (HEX and $1 / \mathrm{N})$. We will examine these patterns further with a richer set of estimations in Section 5 .

Figures 1 to 4 depict all choices by the participants. Each figure has the 146 participants' choices in two paired problems, $\{n-1, n\}$ for $n$ even, indicated by the small markers. (The large markers 
are the predictions of the different behavioral assumptions, explained below.) The horizontal axis in each figure measures a participant's investment in the first asset, $\alpha_{1}^{n}$, of the even-numbered choice problem - the one with correlated assets. The vertical axis uses the investor's choice in the twinned problem $n-1$ and expresses this choice in the action space of problem $n$ : $\widehat{\alpha}_{1}^{n-1}$ is the share that the participant would need to invest in problem $n$ in order to hold the same distribution of earnings that she chooses via her actual choice $\alpha_{1}^{n-1}$ in problem $n-1$. By construction of the twinned-problem design, this share is uniquely determined for each feasible investment in problem $n-1$. Formally, for each $\alpha_{1}^{n-1} \in \mathcal{C}^{n-1}$ there exists a unique $\widehat{\alpha}_{1}^{n-1} \in \mathcal{C}^{n}$ such that

$$
\forall x \in \mathbb{R}: \operatorname{Pr}\left(W^{n-1}(\omega)=x \mid \alpha_{1}^{n-1}\right)=\operatorname{Pr}\left(W^{n}(\omega)=x \mid \alpha_{1}^{n}\right)
$$

and thus each risk that the investor takes on in choice problem $n-1$ can be generated by a possible action in choice problem $n$. By Observation 1, the null hypothesis that participants correctly perceive covariance gives a simple prediction for the figure: under Assumptions A and B (which implicitly prescribe that correlation is fully understood), the two shares $\widehat{\alpha}_{1}^{n-1}$ and $\alpha_{1}^{n}$ are identical and thus the data lie on the 45-degree line. The data are also summarized in terms of mean and standard deviation in Table 2 , second column. ${ }^{14}$

\footnotetext{
${ }^{14}$ For Choice 6 , the prediction of the $1 / \mathrm{N}$ model violates the a lower bound of 40 points for the first-listed asset. In the figures, in Table 2 and all of the subsequent analysis, we therefore set the 1 / N prediction to 40 points.
} 


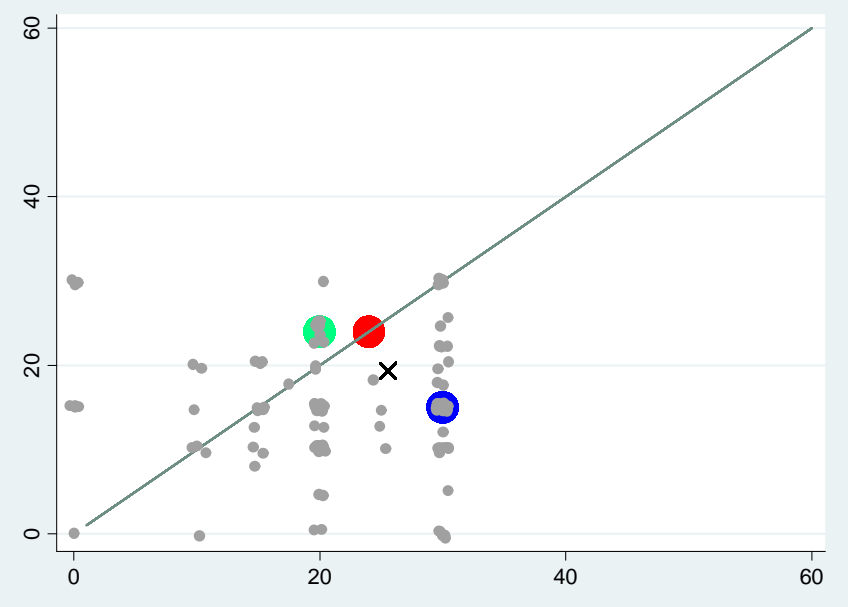

Figure 1: Distribution of investments in Choices 1 and 2. Horizontal axis: $\alpha_{1}^{2}$. Vertical axis: $\widehat{\alpha}_{1}^{1}$. Large $\{$ red, green, blue, $x\}$ markers represent $\{\operatorname{RAT}, \operatorname{HEx}, 1 / \mathrm{N}$, parametric $\}$ predictions.

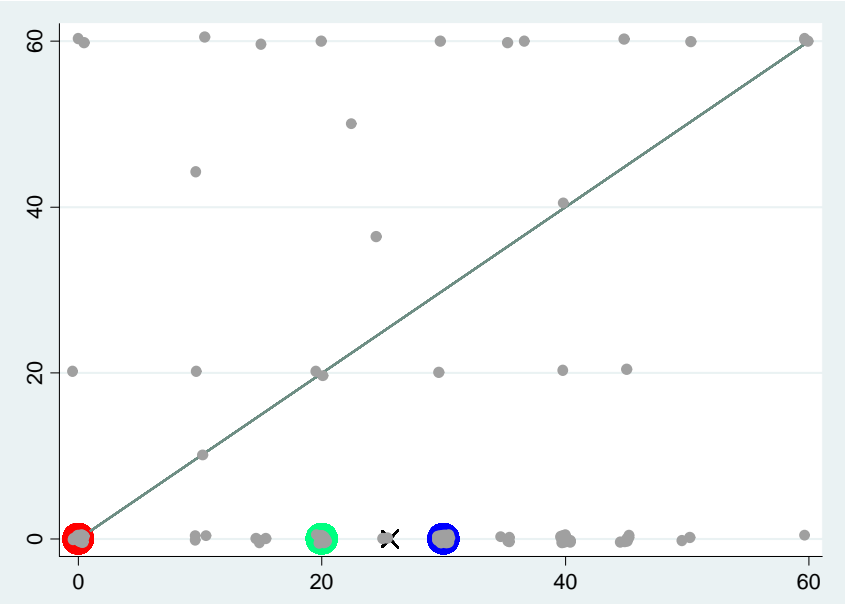

Figure 2: Distribution of investments in Choices 3 and 4. Horizontal axis: $\alpha_{1}^{4}$. Vertical axis: $\widehat{\alpha}_{1}^{3}$. Large $\{$ red, green, blue, $x\}$ markers represent $\{\operatorname{RAT}, \operatorname{HEx}, 1 / \mathrm{N}$, parametric $\}$ predictions. 


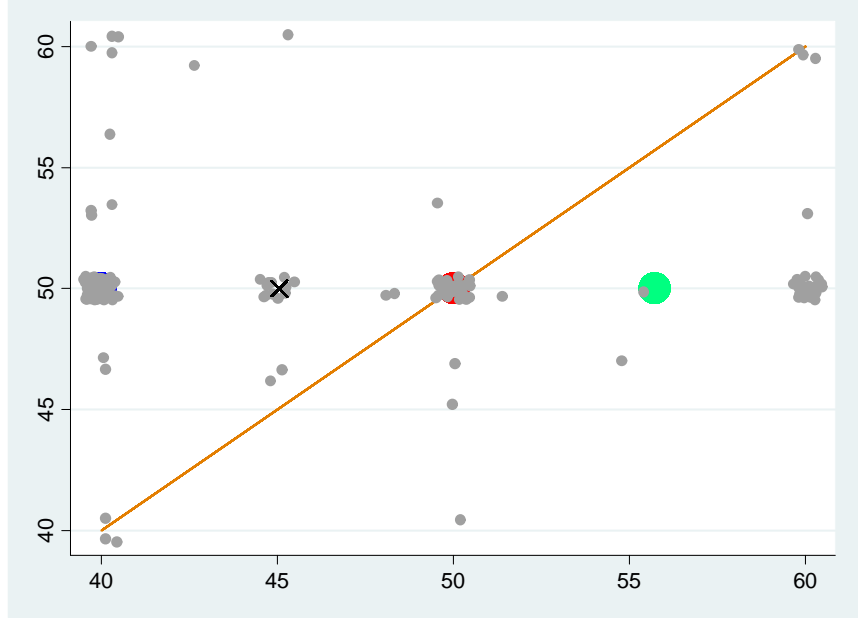

Figure 3: Distribution of investments in Choices 5 and 6 . Horizontal axis: $\alpha_{1}^{6}$. Vertical axis: $\widehat{\alpha}_{1}^{5}$. Large $\{$ red, green, blue, $x\}$ markers represent $\{\mathrm{RAT}, \mathrm{HEx}, 1 / \mathrm{N}$, parametric $\}$ predictions.

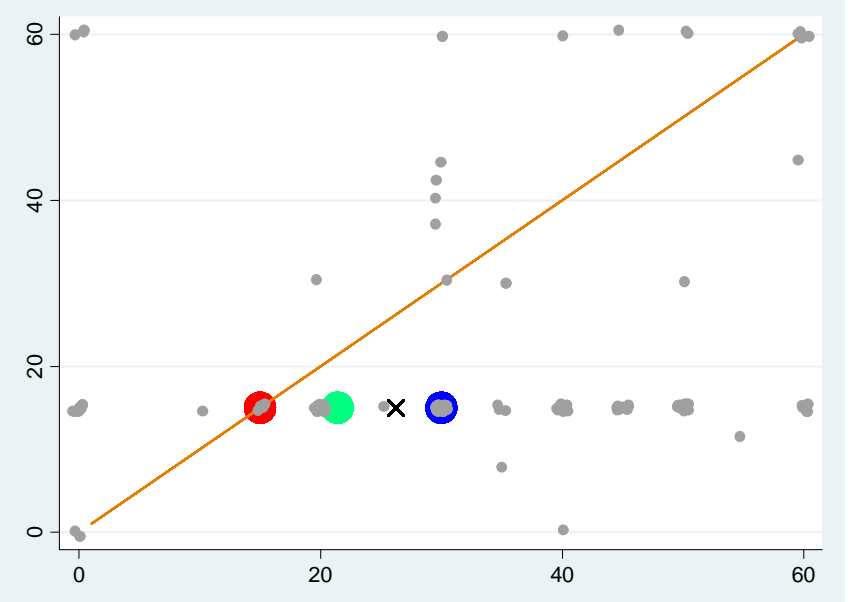

Figure 4: Distribution of investments in Choices 7 and 8. Horizontal axis: $\alpha_{1}^{8}$. Vertical axis: $\widehat{\alpha}_{1}^{7}$. Large $\{$ red, green, blue, $x\}$ markers represent $\{$ RAT, HEX, 1/N, parametric $\}$ predictions. 


\begin{tabular}{ccccc}
\hline \hline$n$ & Data mean (std. dev.) & $\mathrm{RAT}^{n}$ & $\mathrm{HEx}^{n}$ & $(1 / \mathrm{N})^{n}$ \\
\hline 1 & $0.254(.113)$ & 0.4 & 0.4 & 0.25 \\
2 & $0.381(.138)$ & 0.4 & 0.333 & 0.5 \\
3 & $0.126(.306)$ & 0 & 0 & 0 \\
4 & $0.444(.234)$ & 0 & 0.333 & 0.5 \\
5 & $0.839(.053)$ & 0.833 & 0.833 & 0.833 \\
6 & $0.778(.127)$ & 0.833 & 0.929 & 0.667 \\
7 & $0.328(.225)$ & 0.25 & 0.25 & 0.25 \\
8 & $0.545(.267)$ & 0.25 & 0.357 & 0.5 \\
\hline
\end{tabular}

Table 2: Proportions of investment in first-listed asset.

Data and benchmark model predictions, separated

by choice problem

The figures show strong and systematic deviations from the hypothesis that Assumptions A and B hold. Not only do only very few observations lie on the 45-degree lines but there are also patterns in the data that cannot be driven by unsystematic disturbances. Corresponding statistical tests soundly reject the prediction of Assumptions A and B that behavior is unchanged within pairs of twinned problems. For each of the four pairs, Wilcoxon matched-pairs sign-rank tests reject the hypothesis of identical distributions of portfolios at a significance level of 0.001 . This shows that the use of correlated versus uncorrelated asset returns has a significant impact on choices.

As another measure of the accuracy of the null hypothesis of correct perception of correlation we ask how many participants make portfolio choices that are exactly identical between twinned problems. The answer is contained in Table 3 showing that the large majority of the participants never or almost never choose portfolios that are identical between twinned problems. $97.2 \%$ of the participants choose a different set of portfolios between two twinned problems weakly more often than they choose the same portfolio. $60.9 \%$ never choose the same portfolio twice. 


\begin{tabular}{cccc}
\hline \hline$\#$ & Freq. & $\%$ & Cum. \% \\
\hline 0 & 89 & 60.9 & 60.9 \\
1 & 37 & 25.3 & 86.2 \\
2 & 16 & 11.0 & 97.2 \\
3 & 3 & 2.1 & 99.3 \\
4 & 1 & 0.7 & 100.0 \\
\hline Total & 146 & 100.0 & \\
\hline \hline
\end{tabular}

Table 3: Frequencies of choosing identical portfolios in twinned

choice problems (out of 4)

Of course, the deviations may be influenced by different sources and we must to be careful not to interpret random deviations from the 45-degree line as evidence of correlation neglect. We therefore turn to statistical estimations that allow concluding that the deviations from the prediction of Assumptions $\mathrm{A}$ and $\mathrm{B}$ are indeed systematic in the way that we hypothesize. The first such estimation is an OLS regressions that summarizes the statistical connection between the data and extreme predictions Hex and $(1 / \mathrm{N})$. These predictions are indicated in the figures - the green and blue marker, respectively - as well as in columns 4 and 5 of Table 2. As a benchmark prediction, the figures and Table 2 also contain the "rational" prediction RAT of a risk averter who understands the correlation structure (Assumption C). ${ }^{15}$ The dependent variable in the regression is the vertical distance of the data points from the 45-degree line in the figure. The explanatory variables are, analogously, the vertical distances of the two predictions from the 45-degree line, $\widehat{\operatorname{HEX}}^{n-1}-\operatorname{HEX}^{n}$ and $\widehat{1 / \mathrm{N}}^{n-1}-(1 / \mathrm{N})^{n}$, respectively. ${ }^{16}$

\footnotetext{
${ }^{15}$ Where needed to make a unique prediction, the degree of risk aversion is also assumed to be close to zero, as explained in Section 3 (Assumption D).

${ }^{16}$ The estimated model is

$$
\widehat{\alpha}_{1}^{n-1}-\alpha_{1}^{n}=\beta_{2}\left(\widehat{\operatorname{HEX}}^{n-1}-\operatorname{HEX}^{n}\right)+\beta_{3}\left(\widehat{1 / \mathrm{N}}^{n-1}-(1 / \mathrm{N})^{n}\right)+\nu_{i t}
$$

Since the model is differenced we did not include a constant term.
} 


\begin{tabular}{llll}
\hline \hline & $\widehat{\alpha}-\alpha$ & $\widehat{\alpha}-\alpha$ & $\widehat{\alpha}-\alpha$ \\
\hline$\widehat{\operatorname{HEX}}^{n-1}-\mathrm{HEX}^{n}$ & $0.85(.08)^{* * *}$ & - & $0.18(.08)^{* * *}$ \\
$\widehat{1 / \mathrm{N}}^{n-1}-(1 / \mathrm{N})^{n}$ & - & $0.63(.05)^{* * *}$ & $0.56(.04)^{* * *}$ \\
\hline$R^{2}$ & 0.21 & 0.35 & 0.36 \\
$\#$ of obs. & 584 & 584 & 584 \\
\hline \hline
\end{tabular}

Table 4: OLS regression of deviation from 45-degree line on the predicted deviation by two models Hex and 1/N. Standard deviations in parentheses, clustered by subject.

The table shows that both extreme models of correlation neglect are predictive of the data means, as their coefficients are statistically significant both individually (in univariate regressions) and when controlling for the respective other prediction. The $1 / \mathrm{N}$ model has the larger predictive power. The regressions results are also consistent with the data feature that the deviations from the 45-degree line are strong especially when Hex and $1 / \mathrm{N}$ move together. This can be seen by inspection of the models' predictions in Table 2: in Choices 4 and 8, the predictions of HEX and 1/N are both on the same side of the benchmark model RAT. In these two problems the data means also deviate from RAT in the same direction. More generally, the deviations in these problems from the 45-degree line are in the same direction as the deviations of the two stylized models. In contrast, in the two other choice problems, the two stylized models move in opposite directions away from RAT, and the data also conform much more to latter model's prediction.

To see the partial predictive power of all three models, we now consider only correlated tasks (where the three model predictions differ), and regress $\alpha$ on all three model predictions including RAT. 


\begin{tabular}{ccccc}
\hline \hline \multicolumn{5}{c}{$\alpha$} \\
\hline RAT & $-0.44(.07)^{* * *}$ & $0.18(.04)^{* * *}$ & & $-0.13(.06)^{* *}$ \\
HEX & $1.35(.06)^{* * *}$ & - & $0.37(.04)^{* * *}$ & $0.53(.06)^{* * *}$ \\
$1 / \mathrm{N}$ & - & $0.87(.04)^{* * *}$ & $0.65(.05)^{* * *}$ & $0.61(.04)^{* * *}$ \\
\hline$R_{u}^{2}$ & 0.85 & 0.87 & 0.88 & 0.88 \\
$\#$ of obs. & 584 & 584 & 584 & 584 \\
\hline \hline
\end{tabular}

Table 6: OLS regression of investments in even-numbered choice problems on model predictions. Standard deviations in parentheses, clustered by subject. Due to absence of constant, goodness-of-fit is measured by the uncentered $R_{u}^{2}$.

The table shows that when only RAT and 1/N are included, RAT has positive explanatory power and a coefficient of the expected sign. But strikingly, RAT has a negative coefficient once HEX is included. This points again at strong misperceptions of the implied correlation. An important caveat is that the above OLS regressions and Table 2 focus on the data means, implicitly viewing all participants as homogeneous - whereas the data show strong patterns of heterogeneity. Section 5 therefore shows model estimates where heterogeneity between the different participants is allowed.

\section{Estimates of Heterogeneous-Type Models}

In this section we estimate the parameters of the model presented in Section 3 , where $l \geq 0$ measures the degree to which differences between the (co-)variances are perceived and $k \in[0,1]$ measures the extent to which the decision maker considers covariance at all. Using standard portfolio-choice analysis it is straightforward that the prediction of this parametric model is for the agent to choose $\alpha_{1}^{n}(k, l)=\frac{\left(\sigma_{2}^{2}\right)^{l}-k\left|\sigma_{12}\right|^{l} \cdot \operatorname{sgn}\left(\sigma_{12}\right)}{\left(\sigma_{1}^{2}\right)^{l}-\left(\sigma_{2}^{2}\right)^{l}-2 k\left|\sigma_{12}\right|^{l} \cdot \operatorname{sgn}\left(\sigma_{12}\right)}$ as her proportion of wealth invested in the first asset in decision problem $n$. In our first set of estimations we determine the ML-values of $(k, l)$ for each individual separately, from her four decisions that involve correlated assets. ${ }^{17}$

For the estimation we assume that participant $i$, when making her choice $\alpha_{1}^{n, i}$, has her own

\footnotetext{
${ }^{17}$ All estimations in this section involve only Choices 2,4,6 8 as in most odd-numbered tasks, the prediction coincides for all values of $k$ and $l$.
} 


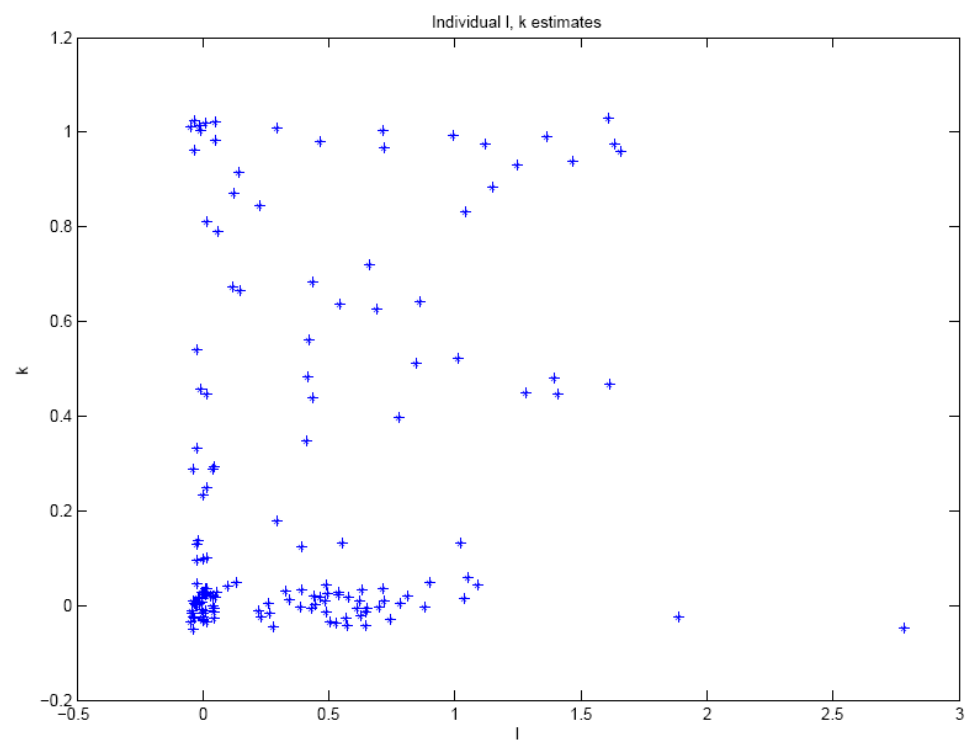

Figure 5: Estimates of parameters $(k, l)$, separately for each of the 146 participants.

individual-specific parameter vector $\left(k^{i}, l^{i}\right)$ and follows the prediction $\alpha_{1}^{n, i}=\alpha_{1}^{n}\left(k^{i}, l^{i}\right)$ but is subject to a disturbance term: she chooses the investment level $60 \cdot\left(\alpha_{1}^{n}\left(k^{i}, l^{i}\right)+\epsilon_{i}^{n}\right)$, rounded to the nearest integer value. We assume that the distribution of $\epsilon_{i}^{n}$ is logistic and i.i.d. across $i$ and $n$. The likelihood of observing the participant's four choice vector $\alpha_{1}^{i}=\left(\alpha_{1}^{2, i}, \alpha_{1}^{4, i}, \alpha_{1}^{6, i}, \alpha_{1}^{8, i}\right)$ is therefore given by

$$
L\left(\alpha_{1}^{i} \mid k^{i}, l^{i}\right)=\prod_{n=2,4,6,8} \operatorname{Pr}\left(\alpha_{1}^{n}\left(k^{i}, l^{i}\right)+\epsilon_{i}^{n}=\alpha_{1}^{n, i}\right)
$$

Maximizing this likelihood separately for each individual gives the distribution of estimates depicted in Figure 5.

The figure suggests the existence of different perceptions of the covariance structure: some participants behave as if they completely ignore all variances and covariance as they have an estimated parameter $l^{i}=0$. Other subjects have "more rational" estimated value of $l^{i}$, but have an estimated $k^{i}$ of zero, indicating that they ignore the off-diagonal elements of the covariance matrix.

To summarize the data patterns more concisely - and enable statistical inference - we now cut down on the number of free parameters and allow the participants to be only of $T$ different types, 
where each type $t \in\{1 \ldots T\}$ has a different parameter vector $\left(k^{t}, l^{t}\right)$. We estimate the proportions of the $T$ types $\left\{\pi^{t}\right\}_{t=1 \ldots T}$ (resticted to sum to one) together with their behavioral parameters $\left\{\left(k^{t}, l^{t}\right)\right\}_{t=1 \ldots T}$. The model is thus a mixture model where the likelihood of observing the collection of participants' choice vectors $\alpha_{1}=\left\{\alpha_{1}^{i}\right\}_{i=1}^{146}$ is:

$$
L\left(\alpha_{1} \mid\left\{k^{t}, l^{t}, \pi^{t}\right\}_{t=1 \ldots T}\right)=\prod_{i=1}^{146} \sum_{t=1}^{T} \pi^{t} \prod_{n=2,4,6,8} \operatorname{Pr}\left(\alpha_{1}^{n}\left(k^{t}, l^{t}\right)+\epsilon_{i}^{n}=\alpha_{1}^{n, i}\right)
$$

The model will generate different results depending on the number of types $T$ that are included. We ran the estimations for up to $T=5$ and report the results for $T \in\{1,2,3\}$ in the follwing Table 5. For $T=4,5$, the results are qualitatively similar to the case $T=3$ but the likelihood is scarcely improved relative to $T=3$.

\begin{tabular}{ccccc}
\hline \hline$T$ & $l l^{*}$ & $\left(l^{1}, k^{1}, \pi^{1}\right)$ & $\left(l^{2}, k^{2}, \pi^{2}\right)$ & $\left(l^{3}, k^{3}, \pi^{3}\right)$ \\
\hline 1 & -2296.6 & $(0.43,0,1)$ & - & - \\
2 & -2288.4 & $(0.39,0,0.91)$ & $(1.1,1,0.09)$ & - \\
3 & -2286.2 & $(0.45,0,0.58)$ & $(1.09,1,0.1)$ & $(0,-, 0.3)$ \\
\hline
\end{tabular}

Table 7: Estimates of parametric mixture model with $T \in\{1,2,3\}$ types.

The table's first row of entries shows the result under the restriction that all participants are of the same type, $T=1$. The estimated parameters indicate that the estimated single type in this model completely ignores the off-diagonal elements of the covariance matrix $\left(k^{1}=0\right)$ and only partially reacts to differences in the assets' variances $\left(l^{1}=0.43\right)$. This is similar to the prediction HEx but includes the ghist of prediction $1 / \mathrm{N}$ by the fact that the sensitivity to variance differences is small. Yet this model is rejected in favor of the two-type model $T=2(\mathrm{p}<0.01$, likelihood ratio test). There, we see that the two co-existant types are quite different in nature: the largest type $\left(\pi^{1}=0.91\right)$ is similar to the type estimated under the $T=1$ model, but the smaller type is much closer to the RAT prediction of fully appreciating all correlation. Finally, under the third model specification with $T=3$, we see that the best-fitting parameter constellation also includes a pure $1 / \mathrm{N}$ type. The result of the $T=3$ estimation is thus not too far from a model where the three archetypical types HEx, RAT and 1/N all appear: each of these three extreme types is reasonably 
well approximated by one of the three estimated types. ${ }^{18}$ However, the $T=2$ specification is not rejected in favor of $T=3(\mathrm{p}=0.221)$.

In sum, he most reliably estimated type is thus the one in the $T=1$ model, showing a considerable prevalence of correlation neglectors in the participant population of our experiment. The prediction of the $T=1$ model is indicated by the x-marker in Figures 1 through 4 . A comparison to Table shows that the prediction of this model is reasonably close to the data mean in each case.

\section{Additional Demonstrations of Correlation Neglect}

Two other sets of experimental tasks are given to the participants with the aim of demonstrating correlation neglect in other ways than in the previous sections. Different subsets of participants received different instructions for these additional tasks. As noted earlier, all of the additional tasks appear after the main part of the experiment and thus the differences in instructions cannot affect the results discussed in the previous sections.

A notable modification from the previously discussed problems is that the expected return varies across assets. Yet all additional tasks are portfolio-choice problems of the same general format as in the experiment's main part. Only very brief additional instructions are therefore needed between the two parts of the experiment. Section 6.1 describes the first set of additional tasks, presented to three different subgroups of particpants after the main experiment. Section 6.2 describes the second set of tasks, presented again to other subgroups of participants. For the task described in Section 6.2 a set of new participants were used, as described there.

\subsection{Falsely Perceived Stochastic Dominance}

\footnotetext{
${ }^{18}$ However, if we estimate a model where the three extreme types HEX, RAT and 1/N are literally assumed to coexist, the proportions are fairly different from those estimated in Table 5. Rather the result are reminiscent of the linear regression in Table 4: the $1 / \mathrm{N}$ type gets weight $\pi^{1 / n}=0.75$ and the Hex type gets only $\pi^{H e x}=0.16$.This shows that the first-listed type estimate in Table 5 , which is similar to Hex, would be much less accurate if $l=1$ was assumed instead of leaving $l$ unrestricted.
} 
The first demonstration is an example involving two assets $U$ and $V$, each with three possible return realizations that occur equi-probably. Differing from the previous continuous-choice problems, the decision-maker here chooses discretely between two portfolios with strictly positive weights of the two assets. The distributions and available portfoilios are designed such that if the decision-maker ignores covariance, like the hexed agent of Section 3 (Assumptions B-E) then she falsely perceives a first-order stochastic dominance relation between the two available portfolios.

\begin{tabular}{cccc} 
& {$[1]$} & {$[2]$} & {$[3]$} \\
\hline$U$ & 30 & 20 & 12 \\
$V$ & 10 & 12 & 30
\end{tabular}

Please indicate your preferred investment, by ticking the box:

I invest 52 points in Asset $U$ and 8 points in Asset $V$.

I invest 26 points in Asset $U$ and 34 points in Asset $V$.

It is obvious that $U$ has a higher return distribution than $V$, with a difference in means of $\frac{10}{3}$. This makes the first choice option relatively attractive due to its high weight on $U$. On the other hand, the negative correlation between the assets makes it worthwhile for a sufficiently risk averse decision-maker to take the safer second choice option.

Crucially, the task is designed such that a hexed decision-maker who ignores the negative correlation would never choose the safe option. It is straightforward to show that if the two assets are perceived to be independent - in the notation of Section 3, if the preferences relation $\succeq$ is defined over $\widehat{\mathcal{W}}$ (Assumption E) and the decision-maker considers assets $\widehat{U}, \widehat{V}$ instead of $U, V$ - then the first choice option first-order stochastically dominates the second choice option.

The task was presented to 30 subjects, 29 of which (97\%) chose the first option. This result is consistent with the presence of hexed decision-makers. However, it may also be driven by rational preference maximization-perhaps all of these 29 participants correctly perceived the covariance structure but are not sufficiently risk averse to avoid the relatively higher risk. To demonstrate 
the effect of the perception of the covariance structure, we therefore repeat the task with 39 different participants, under a presentation frame where the covariance need not be considered by the participants.

\begin{tabular}{cccc} 
& {$[1]$} & {$[2]$} & {$[3]$} \\
\hline$U^{\prime}$ & 27.3 & 18.9 & 14.4 \\
$V^{\prime}$ & 15.5 & 18.7 & 22.2
\end{tabular}

Please indicate your preferred investment, by ticking the box:

I invest 60 points in Asset $U^{\prime}$ and 0 points in Asset $V^{\prime}$.

I invest 0 points in Asset $U^{\prime}$ and 60 points in Asset $V^{\prime}$.

The reader can verify that the return distributions of assets $U^{\prime}$ and $V^{\prime}$ are identical to the distributions resulting from the two choice options in the first problem, involving assets $U$ and $V$ (up to rounding error). Therefore the two choice options in the second problem, allowing only investments of all wealth in one asset, are identical to the options in the first problem - a pure framing variation. Since no diversification opportunities are offered, covariance between the available options is irrelevant in the second problem and even hexed decision-makers would not make a mistake. The pair of problems therefore gives rise to the possibility of preference reversals for hexed agents. Indeed we see a significant change in behavior: in the second framing variation of the problem, only 20 out of 39 participants (51\%) choose the fist option. ${ }^{19}$ The difference between

\footnotetext{
${ }^{19}$ The result is further corroborated by additional framing variations where only one of the two choice options of the Choice involving Assets $U$ and $V$ is replaced. 34 subjects (all of whom are included in the analysis of Sections 2-5 but who are not among the subjects discssed previously in Section 6) faced the same binary task twice, in two different framing variations that are difficult to recognize as being identical. In the first framing variation that they saw, the first choice option (52 points in $U$ and 8 points in $V$ ) was shown as the option of investing everything in $U^{\prime}$. In the second variation, the second option (26 points in $U$ and 34 points in $V$ ) was replaced by the option of investing everything in $V^{\prime}$. Under both framing variations, correlation neglect is irrelevant and behavior of a hexed agent should be identical to the case where both choice options are shown as $U^{\prime}$ and $V^{\prime}$. Indeed, 18 out of 33 participants (55\%) opt for the first choice option under the first framing variation (one participant did not fill in the decision sheet) and 16 out of 34 particpants under the second framing variation.
} 
results in the two framing variations is significant at $\mathrm{p}<0.01$ (Wilcoxon two-sample test).

\subsection{Ignorance of Abitrage}

The second demonstration consists of two portfolio-choice problems that use three assets per problem. Each problem is designed such that there exist portfolios combining two assets that statewise dominate the third asset. Any investment in the third asset is therefore a violation of arbitrage freeness. These two tasks are presented at the end of the experiment (like those in Section 6.1) to 40 participants of the main experiment described in Section 2 and to 96 new participants. The 96 new participants are first presented with the same instructions and tasks as in the main experiment, with the sole difference that in the four problems involving correlated assets (problems 2, 4,6,8) their choice options are restricted to two portfolios. (The results of these restricted tasks essentially confirm the results of the previous sections and we skip them for brevity.) In total there are 136 participants completing the two tasks described here.

11. Invest each of your 60 points in Asset U", Asset V" or Asset W", as given below.

\begin{tabular}{cccc} 
& {$[1]$} & {$[2]$} & {$[3]$} \\
\hline$U^{\prime \prime}$ & 15 & 38 & 7 \\
$V^{\prime \prime}$ & 39 & 5 & 16 \\
$W^{\prime \prime}$ & 20 & 15 & 13
\end{tabular}

In the first of the two additional tasks, participants choose their portfolio weights on Assets $U^{\prime \prime}, V^{\prime \prime}$ and $W^{\prime \prime}$, as depicted above. ${ }^{20}$ One can check easily that asset $W^{\prime \prime}$ is dominated by a continuum of combinations of $U^{\prime \prime}$ and $V^{\prime \prime}$ - in particular it is dominated by $\frac{1}{3} U^{\prime \prime}+\frac{2}{3} V^{\prime \prime}$ (with a expected return difference of 4 Euros - not even a small difference). This arbitrage is due to the negative correlation of $U^{\prime \prime}$ and $V^{\prime \prime}$ that allows an effective insurance against their respective lowreturn outcomes. But many participants appear to neglect the hedging opportunity: the average portfolio weight of $W^{\prime \prime}$ is 0.257 , close to the uniform weight. (The average weights of $U^{\prime \prime}$ and $V^{\prime \prime}$ are 0.346 and 0.397 respectively.) Moreover, the distribution of investments reveals that 84 out of

\footnotetext{
${ }^{20}$ In the experiment the asset labels are $U, V$ and $W$ (symbols that we already used for other participants' problems).
} 
136 participants (75\%) invested a strictly positive wealth share in $W^{\prime \prime}$. That is, the large majority of subjects makes a dominated choice in this task.

The last-mentioned result is even more pronounced in the second additional task:

12. Invest each of your 60 points in Asset X, Asset Y or Asset Z, as given below.

\begin{tabular}{cccc} 
& {$[1]$} & {$[2]$} & {$[3]$} \\
\hline$X$ & 14 & 22 & 12 \\
$Y$ & 27 & 5 & 16 \\
$Z$ & 18 & 16 & 13
\end{tabular}

Here, Asset $Z$ is dominated by a continuum of combinations of $X$ and $Y$ - e.g., by $\frac{2}{3} X+\frac{1}{3} Y$. Only 19 out of 136 participants (14\%) invested nothing in $Z$ and thereby avoided the dominated investment. The remaining $86 \%$ of subjects appear not to appreciate the dominance relation that arises due to negative correlation of $X$ and $Y$. The average portfolio weights of $X, Y$ and $Z$ are $0.399,0.308$ and 0.293 respectively.

\section{Conclusion}

Our experiments provide strong evidence that people neglect correlation when making financial decisions. Partly, this may derive from the form of $1 / n$ rule that Benartzi and Thaler (2001) observe in financial decision making that also has been found in other contexts. Simonson (1990) as well as Read and Loewenstein (1995) have suggested that people use strategies of "naive diversification" by instinctively diversifying their choices despite underlying preferences that do not warrant such diversification. For instance, a treak-or-treater who prefers Mars bars to Snickers may too frequently choose Snickers over Mars so as not to end up with an unbalanced candy supply. Indeed, restaurantgoers often seem reluctant to order dishes that have previously been ordered, even when they know that they will not share dishes.

From a modeling perspective, however, in settings where people's choice sets imperfectly observed down, the $1 / n$ heuristic does not provide the researcher with much guidance as to how people 
will behave. In addition to predicting settings where people under-diversify, our model of correlation neglect adds to the $1 / n$ heuristic by predicting how people's choices depart systematically from what they would choose had they taken correlation into account fully. Proposition 1 demonstrates in the context of a two-asset model that when correlation is positive, correlation neglectors diversify more than standard theory predicts - exaggerating its benefits - while when correlation is negative they invest too little.

People who systematically underappreciate correlation will be willing to pay less than they should for assets that hedge the risk in their portfolio and willing to pay more than they should for assets that magnify the risk in their portfolio. Shleifer (2000) outlines several severe impediments to arbitrage on financial markets, from risk to transaction costs. Without perfect arbitrage, a systematic underappreciation of correlation may cause $\beta$ to be underpriced.

\section{References}

Benartzi, S. and Thaler, R.: 2001, Naive diversification strategies in defined contribution saving plans, American Economic Review 91(1), 79-98.

Brunnermeier, M.: 2009, Deciphering the liquidity and credit crunch 2007-2008, Journal of Economic Perspectives 23(1), 77-100.

Gubaydullina, Z. and Spiwoks, M.: 2009, Portfolio diversification: an experimental study.

Hellwig, M.: 2009, Systemic risk in the financial sector: An analysis of the subprime-mortgage financial crisis, De Economist 157(2), 129-207.

Kahneman, D. and Tversky, A.: 1979, Prospect theory: An analysis of decision under risk, Econometrica 47(2), 263-292.

Kallir, I. and Sonsino, D.: 2009, The neglect of correlation in allocation decisions, Southern Economic Journal 75(4), 1045-1045.

Kroll, Y., Levy, H. and Rapoport, A.: 1988, Experimental tests of the separation theorem and the capital asset pricing model, American Economic Review 78(3), 500-519. 
Polkovnichenko, V.: 2005, Household portfolio diversification: A case for rank-dependent preferences, Review of Financial Studies 18(4), 1467-1502.

Rabin, M. and Weizsäcker, G.: 2009, Narrow bracketing and dominated choices, American Economic Review 99(4), 1508-1543.

Read, D. and Loewenstein, G.: 1995, Diversification bias: Explaining the discrepancy in variety seeking between combined and separated choices, Journal of Experimental Psychology: Applied $\mathbf{1}(1), 34-49$.

Shleifer, A.: 2000, Inefficient markets: an introduction to behavioral finance, Oxford University Press.

Simonson, I.: 1990, The effect of purchase quantity and timing on variety seeking behavior, Journal of Marketing Research 32, 150-162.

Tversky, A. and Kahneman, D.: 1981, The framing of decisions and the psychology of choice, Science 211(4481), 453-458.

\section{Appendix A: Instructions}

\section{Welcome!}

You are about to participate in an experiment in decision making. Universities and research agencies have provided the funds for this experiment.

In this experiment we will first ask you to read instructions that explain the decision scenarios you will be faced with. We will also ask you to answer questions that test your understanding of what you read. Finally, you will be asked to make decisions that will allow you to earn money. Your monetary earnings will be determined by your decisions and by chance. All that you earn is yours to keep and will be paid to you in private, in cash, after today's session.

Only for coming here and completing the experiment, you will also receive a fixed participation fee of EUR 5.00. The earnings that you make during the experiment will be added to this amount. 
It is important to us that you remain silent and do not look at other people's work. If you have any questions or need assistance of any kind, please raise your hand, and an experimenter will come to you. If you talk, exclaim out loud, etc., you will be asked to leave and will forfeit your earnings. Thank you.

The determination of your pay-out

We ask you to make 11 decisions in total during this experiment. Of these 11 decisions, only one will be actually paid out. This will be determined by a random draw at the end of today's session, when you will make a draw from a stack of 11 cards. The card that you draw will determine which decision will be paid out to you. Every participant in the room will make a separate draw, so different decisions will be paid out to different participants.

\section{Description of the decisions}

All 11 decisions follow essentially the same format. In each of them, you will allocate 60 points among two different assets. An asset is an investment opportunity that yields a return. The asset returns are probabilistic, meaning that at the time you invest you cannot be sure how much return an asset yields. Each asset has at most 4 different return values. For example, a given asset may have the following possible return values, in Euros:

Asset A: 10281223

If you invest all 60 points in this asset, you would receive the full return that is indicated by the return values. In this example, if you invest all points in Asset A, you would either receive $€ 10$ or $€ 28$ or $€ 12$ or $€ 23$. You would not know which of the 4 possible returns you would get.

In each decision, there are two available assets that have different distributions of the probabilistic return, and you can allocate your 60 points between the two assets in the way that suits you best. If you invest less than the full 60 points in a given asset, you receive proportionally less than the full return. For example, if you invest 30 points in Asset A, you would receive either $€ 5$ or $€ 14$ or $€ 6$ or $€ 11.50$ from Asset A, plus another probabilistic amount from investing the remaining 30 points in the second asset.

For both assets, the actual return depends on the value of a single random outcome. There are 4 possible values of this outcome, and the return of each asset is different for the 4 different 
outcome values. The four possible values of the outcome all have the same probability of $1 / 4$. We implement this by flipping 2 coins. One of our two coins has labels \# and \& on its two sides. The other coin has two labels ${ }^{*}$ and ${ }^{\wedge}$. When we flip both of the two coins, there are 4 possible combinations of labels that can come up:

$$
\#^{*} \#^{\wedge} \&^{*} \&^{\wedge}
$$

Each of these four outcomes has the exact same probability of $1 / 4$ (or 25\%). For both of the assets, the outcome depends on which combination of labels comes up. We will toss the two coins only once at the end of the experiment, and this double coin toss will determine the return of both assets in each of the 11 decisions.

For example, one decision that you may face could look like this:

Invest each of your 60 points in either Asset A or Asset B, as given below.

$$
\#^{*} \#^{\wedge} \&^{*} \&^{\wedge}
$$

Asset A: 15301520

$$
\#^{*} \#^{\wedge} \&^{*} \&^{\wedge}
$$

Asset B: 10203010

In this example, how much you earn depends on how many points you invest in Asset A and Asset $\mathrm{B}$ and on the outcome of the two coin tosses. For instance, if you invest 20 points in Asset A and 40 points in Asset B - i.e. you invest one-third of your points in Asset A and two-thirds in Asset B - and if the value of the random outcome is $\&^{*}$, then your overall earnings would be:

$$
(1 / 3) \boldsymbol{\square} € 15+(2 / 3) \boldsymbol{\square} € 30=€ 25
$$

Please note that you would be paid this amount only if this particular decision is paid out. In the actual experiment, only one of the 11 decisions will be paid out, as described above.

Also, importantly, note again that only one random outcome is drawn (here, $\&^{*}$ ), which determines the return of both assets. 
You can easily calculate other possible earnings, for different combinations of token allocations and random outcomes. For example, if you invest 48 points in Asset A and 12 in Asset B - hence, four-fifths in Asset $\mathrm{A}$ - and if the random outcome value is $\#^{\wedge}$, your return is:

$$
(4 / 5) \boldsymbol{\square} € 30+(1 / 5) \boldsymbol{\square} € 20=€ 28
$$

Or, if you choose the same division (48/12 in Assets A/B) but the random outcome value is $\#^{*}$, your return is:

$$
(4 / 5) \mathbf{\square} € 15+(1 / 5) \mathbf{\square} € 10=€ 14
$$

The 11 decisions differ from one another as well as from the above example in two ways:

Firstly, the probabilistic returns of the assets are different. (But they all have at most 4 possible values.)

Secondly, not all investment combinations are available in each case. For example, some decisions require you to invest at least some minimum amount in a given asset. This requirement appears clearly in the instruction line of each separate decision. For example, the precise wording of the instruction may be as follows.

Invest each of your 60 points in either Asset A or Asset B, as given below.

Note: At least 20 points must be invested in Asset A.

$$
\#^{*} \#^{\wedge} \&^{*} \&^{\wedge}
$$

Asset A: 15201530 Investment: points $(\mathbf{\square} 20)$

$$
\#^{*} \#^{\wedge} \&^{*} \&^{\wedge}
$$

Asset B: 1003010 Investment: ___ points

Please ensure that your two investments sum to 60 points.

For all decisions, only positive token investments are feasible. That is, at least 0 points must be invested in each asset in any case, even if this requirement is not explicitly mentioned in the respective instruction lines.

Before proceeding to the actual decisions, we will ask you to complete an understanding test of the instructions. 
Please wait until the understanding test is distributed. If you have any questions about the instructions up to here, please raise your hand.

Understanding test

Please record your code number on this sheet, as well as on all subsequent sheets during the experiment

Consider the following investment decision and answer the questions (1) to (3) below. You will only be allowed to continue with the experiment after answering correctly. If you have a question of any kind, please raise your hand.

Invest each of your 60 points in either Asset A or Asset B, as given below.

Note: At least 10 points must be invested in Asset A.

$$
\#^{*} \#^{\wedge} \&^{*} \&^{\wedge}
$$

Asset A: 30201510 Investment: points

$$
\#^{*} \#^{\wedge} \&^{*} \&^{\wedge}
$$

Asset B: 400530 Investment: points

Please ensure that your two investments sum to 60 points.

Questions:

(1) Suppose that this decision is paid to you at the end of the experiment and that you invest 60 points in Asset $\mathrm{A}$ and 0 points in Asset B. If the random outcome value is $\#^{\wedge}$, what do you earn? EUR

(2) Suppose that this decision is paid to you at the end of the experiment and that you invest 15 points in Asset A and 45 points in Asset B. If the random outcome value is \#^, what do you earn? EUR

(3) Suppose that this decision is paid to you at the end of the experiment and that you invest 30 points in Asset A and 30 points in Asset B. If the random outcome value is \&*, what do you earn? EUR

Once you finish the understanding test, please wait for instructions for the decisions. If you have a question, please raise your hand. Please make sure that the code number is recorded on the understanding test. 


\section{The 11 decisions}

In each of the following decisions, please make sure that your investments exactly add up to 60 points. If they do not add up to 60 in the task that is to be paid out, you will only be paid the smallest possible pay-out in the respective decision.

For decisions 1-10, please indicate your investments next to the respective assets.

1.

Invest each of your 60 points in either Asset A or Asset B, as given below.

$$
\#^{*} \#^{\wedge} \&^{*} \&^{\wedge}
$$

Asset A: 15211521 Investment: points

$$
\#^{*} \#^{\wedge} \&^{*} \&^{\wedge}
$$

Asset B: 12122424 Investment: points

Please ensure that the two investments add up to 60 points.

2.

Invest each of your 60 points in either Asset C or Asset D, as given below.

Note: At least 30 points must be invested in Asset D.

$$
\#^{*} \#^{\wedge} \&^{*} \&^{\wedge}
$$

Asset C: 1830618 Investment: points

$$
\#^{*} \#^{\wedge} \&^{*} \&^{\wedge}
$$

Asset D: 12122424 Investment: points

Please ensure that your two investments sum to 60 .

3.

Invest each of your 60 points in either Asset E or Asset F, as given below.

$$
\#^{*} \#^{\wedge} \&^{*} \&^{\wedge}
$$

Asset E: 12241224 Investment: points

$$
\#^{*} \#^{\wedge} \&^{*} \&^{\wedge}
$$

Asset F: 12122424 Investment: points

Please ensure that the two investments add up to 60 points. 
4.

Invest each of your 60 points in either Asset G or Asset H, as given below.

$$
\#^{*} \#^{\wedge} \&^{*} \&^{\wedge}
$$

Asset G: 12241224 Investment: points

$$
\#^{*} \#^{\wedge} \&^{*} \&^{\wedge}
$$

Asset H: 12181824 Investment: points

Please ensure that the two investments add up to 60 points.

5.

Invest each of your 60 points in either Asset I or Asset J, as given below.

$$
\#^{*} \#^{\wedge} \&^{*} \&^{\wedge}
$$

Asset I: 14211421 Investment: points

$$
\#^{*} \#^{\wedge} \&^{*} \&^{\wedge}
$$

Asset J: 14142121 Investment: points

Please ensure that the two investments add up to 60 points.

6.

Invest each of your 60 points in either Asset K or Asset L, as given below. Note: At least 40 points must be invested in Asset K.

$$
\#^{*} \#^{\wedge} \&^{*} \&^{\wedge}
$$

Asset K: 14211421 Investment: points

$$
\#^{*} \#^{\wedge} \&^{*} \&^{\wedge}
$$

Asset L: 1403521 Investment: points

Please ensure that the two investments add up to 60 points.

7.

Invest each of your 60 points in either Asset M or Asset N, as given below.

$$
\#^{*} \#^{\wedge} \&^{*} \&^{\wedge}
$$


Asset M: 12301230 Investment: points

$$
\#^{*} \#^{\wedge} \&^{*} \&^{\wedge}
$$

Asset N: 12123030 Investment: points

Please ensure that the two investments add up to 60 points.

8.

Invest each of your 60 points in either Asset $\mathrm{O}$ or Asset $\mathrm{P}$, as given below.

$$
\#^{*} \#^{\wedge} \&^{*} \&^{\wedge}
$$

Asset O: 12301230 Investment: points

$$
\#^{*} \#^{\wedge} \&^{*} \&^{\wedge}
$$

Asset P: 12182430 Investment: points

Please ensure that the two investments add up to 60 points.

9.

Invest each of your 60 points in either Asset $\mathrm{Q}$ or Asset R, as given below.

$$
\#^{*} \#^{\wedge} \&^{*} \&^{\wedge}
$$

Asset Q: 16152021 Investment: points

$$
\#^{*} \#^{\wedge} \&^{*} \&^{\wedge}
$$

Asset R: 14151918 Investment: points

Please ensure that the two investments add up to 60 points.

10.

Invest each of your 60 points in either Asset $\mathrm{S}$ or Asset $\mathrm{T}$, as given below.

$$
\#^{*} \#^{\wedge} \&^{*} \&^{\wedge}
$$

Asset S: 18171918 Investment: points

$$
\#^{*} \#^{\wedge} \&^{*} \&^{\wedge}
$$

Asset T: 16161717 Investment: points

Please ensure that the two investments add up to 60 points. 
[New set of instructions.]

Comment on decision 11: For this decision, you only have the choice between two different combinations of token investments. The two available options will be indicated below the description of the assets.

A further change is that only 3 random outcomes are possible, not 4 . Correspondingly, each asset has only 3 possible return values. If you choose the card with number 11, indicating that decision 11 is paid out to you, then we simply ignore the fourth possible outcome of the double coin toss, when determining your pay-out. That is, if the double coin toss yields $\&^{\wedge}$, then we will repeat the double coin toss until we get a result of $\#^{*}$ or $\#^{\wedge}$ or $\&^{*}$. The pay-out will then follow the realized outcome. The three outcomes $\#^{*}, \#^{\wedge}$ and $\&^{*}$ are therefore the only relevant outcomes in this decision, and they are still equally likely to appear. Effectively, each of the three outcomes will result with probability $1 / 3$, or one out of three times.

11.

Invest each of your 60 points in either Asset U or Asset V, as given below. Indicate your investment below the description of the assets, choosing from the two available investments.

$$
\#^{*} \#^{\wedge} \&^{*}
$$

Asset U: 302012

$$
\#^{*} \#^{\wedge} \&^{*}
$$

Asset V: 101230

Please indicate your preferred investment, by ticking the box:

- I invest 52 points in Asset $\mathrm{U}$ and 8 points in Asset V.

- I invest 26 points in Asset $\mathrm{U}$ and 34 points in Asset V.

Once you finish making the decisions, please wait until the experimenter collects the decision sheets. If you have a question, please raise your hand. Please make sure that the code number is recorded on the first decision sheet. 


\section{Appendix B: Further result on ignored dominance relation} $[\mathrm{tbc}]$

\section{Appendix C: Proof}

Proof of Proposition 1 Let

$$
V=\left(\begin{array}{cc}
\sigma_{1}^{2} & k \sigma_{12} \\
k \sigma_{12} & \sigma_{2}^{2}
\end{array}\right) .
$$

At an interior solution, $\alpha_{1}=\frac{\sigma_{2}^{2}-k \sigma_{12}}{\sigma_{1}^{2}+\sigma_{2}^{2}-2 k \sigma_{12}}$, so

$$
\begin{aligned}
\operatorname{sgn}\left(\frac{\partial \alpha_{1}}{\partial k}\right) & =\operatorname{sgn}\left(-\sigma_{12}\left(\sigma_{1}^{2}+\sigma_{2}^{2}-2 k \sigma_{12}\right)+2 \sigma_{12}\left(\sigma_{2}^{2}-k \sigma_{12}\right)\right) \\
& =\operatorname{sgn}\left(\sigma_{12}\left(\sigma_{2}^{2}-\sigma_{1}^{2}\right)\right) .
\end{aligned}
$$

Wlog assume that $\sigma_{2}^{2}-\sigma_{1}^{2} \geq 0$ so that $\alpha_{1} \geq \frac{1}{2}$. When $\sigma_{12}>0, \alpha_{1}$ is increasing in $k$, meaning that a hexed investor with $k=0$ chooses a portfolio closer to 50-50 than a rataional agent. Conversely, when $\sigma_{12}<0, \alpha_{1}$ is decreasing in $k$, meaning that a hexer with $k=0$ chooses a portfolio further from 50-50 than a rational agent. Q.E.D. 